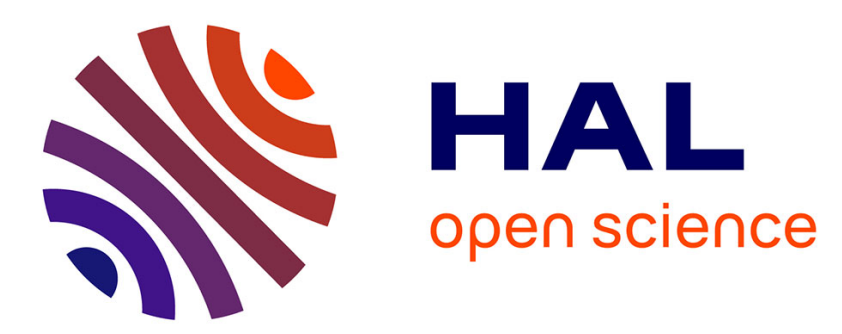

\title{
Theory for electron transfer from a mixed-valence dimer with paramagnetic sites to a mononuclear acceptor
}

\author{
E.L. Bominaar, C. Achim, S.A. Borshch
}

\section{To cite this version:}

E.L. Bominaar, C. Achim, S.A. Borshch. Theory for electron transfer from a mixed-valence dimer with paramagnetic sites to a mononuclear acceptor. Journal of Chemical Physics, 1999, 110, pp.1141111422. 10.1063/1.479082. hal-00006809

\section{HAL Id: hal-00006809 \\ https://hal.science/hal-00006809}

Submitted on 25 May 2021

HAL is a multi-disciplinary open access archive for the deposit and dissemination of scientific research documents, whether they are published or not. The documents may come from teaching and research institutions in France or abroad, or from public or private research centers.
L'archive ouverte pluridisciplinaire HAL, est destinée au dépôt et à la diffusion de documents scientifiques de niveau recherche, publiés ou non, émanant des établissements d'enseignement et de recherche français ou étrangers, des laboratoires publics ou privés. 


\title{
Theory for electron transfer from a mixed-valence dimer with paramagnetic sites to a mononuclear acceptor
}

\author{
E. L. Bominaara) and C. Achim ${ }^{\text {b) }}$ \\ Department of Chemistry, Carnegie Mellon University, Pittsburgh, Pennsylvania 15213 \\ S. A. Borshch ${ }^{\mathrm{c})}$ \\ Institut de Recherches sur la Catalyse, CNRS, 69626 Villeurbanne cedex, France
}

Polynuclear transition-metal complexes, such as $\mathrm{Fe}-\mathrm{S}$ clusters, are the prosthetic groups in a large number of metalloproteins and serve as temporary electron storage units in a number of important redox-based biological processes. Polynuclearity distinguishes clusters from mononuclear centers and confers upon them unique properties, such as spin ordering and the presence of thermally accessible excited spin states in clusters with paramagnetic sites, and fractional valencies in clusters of the mixed-valence type. In an earlier study we presented an effective-mode (EM) analysis of electron transfer from a binuclear mixed-valence donor with paramagnetic sites to a mononuclear acceptor which revealed that the cluster-specific attributes have an important impact on the kinetics of long-range electron transfer. In the present study, the validity of these results is tested in the framework of more detailed theories which we have termed the multimode semiclassical (SC) model and the quantum-mechanical (QM) model. It is found that the qualitative trends in the rate constant are the same in all treatments and that the semiclassical models provide a good approximation of the more rigorous quantum-mechanical description of electron transfer under physiologically relevant conditions. In particular, the present results corroborate the importance of electron transfer via excited spin states in reactions with a low driving force and justify the use of semiclassical theory in cases in which the QM model is computationally too demanding. We consider cases in which either one or two donor sites of a dimer are electronically coupled to the acceptor. In the case of multiconnectivity, the rate constant for electron transfer from a valence-delocalized (class-III) donor is nonadditive with respect to transfer from individual metal sites of the donor and undergoes an order-of-magnitude change by reversing the sign of the intradimer metal-metal resonance parameter $(\beta)$. In the case of single connectivity, the rate constant for electron transfer from a valence-localized (class-II) donor can readily be tuned over several orders of magnitude by introducing differences in the electronic potentials at the two metal sites of the donor. These results indicate that theories of cluster-based electron transfer, in order to be realistic, need to consider both intrinsic electronic structure and extrinsic interactions of the cluster with the protein environment.

\section{INTRODUCTION}

Systems with unbalanced spatial electron distributions strive toward thermodynamic equilibrium by means of electron transfer from electron-rich regions to electron-poor areas. This phenomenon, observed in a wide range of organic and inorganic substances, plays a vital role in living organisms. ${ }^{1}$ For example, terrestrial life is driven by the combustion of hydrocarbons, a process controlled by a complex sequence of electron-transfer reactions. Various prosthetic groups, tethered to proteins, have been identified as donor and acceptor sites for biological electron transfer. ${ }^{2}$ These systems include organic groups, such as flavins and inorganic electron-storage units. The latter units can be subdivided into mononuclear sites and polynuclear clusters

${ }^{a)}$ Author to whom correspondence should be addressed; electronic mail: eb7g@andrew.cmu.edu

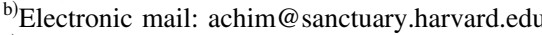

c) Author to whom correspondence should be addressed; electronic mail: borchtch@catalyse.univ-lyon1.fr which are exemplified by metalloporphyrins and iron-sulfur clusters, respectively. ${ }^{3,4}$ The donor and acceptor in biological electron transfer can either belong to the same protein or be located in different protein moieties. ${ }^{5}$ In the latter case, the formation of a protein complex is required for electron transfer to take place. Intraprotein and interprotein electrontransfer reactions occur, for example, in the catalytic cycle of nitrogenase, the two-component metalloenzyme that catalyses the six-electron reduction of molecular nitrogen to ammonia. ${ }^{6}$

Factors determining the kinetics of electron transfer in biological systems are the subject of continuing interest. ${ }^{7}$ The rate constant for a nonadiabatic (long-range) electrontransfer reaction can be expressed as $k_{\text {et }}$ $=\pi^{1 / 2} \hbar^{-1} H_{\mathrm{DA}}^{2}\left(\chi k_{B} T\right)^{-1 / 2} \exp \left(-\Delta G^{*} / k_{B} T\right)$ in semiclassical approximation, where $\Delta G^{*}=\left(\chi+\Delta G^{0}\right)^{2} / 4 \chi$ is the free energy of activation, $\chi$ is the reorganization parameter, $\Delta G^{0}$ is the free-energy change for the electron-transfer reaction, and $H_{\mathrm{DA}}$ is the electronic matrix element that couples the donor 
and acceptor. ${ }^{8}$ It is a well-established fact that electronic coupling is mediated by the protein and that without this medium the tunneling rate over the donor-acceptor distances encountered in biological electron transfer would be vanishingly small. ${ }^{9,10}$ Kinetics studies of photoinduced electron transfer in photoreaction centers have revealed that $H_{\mathrm{DA}}$ decays exponentially with donor-acceptor distance. ${ }^{9,11}$ This result is consistent with the notion that the electronic coupling is propagated through the protein, e.g., by a superexchange mechanism. ${ }^{12,13}$ However, whether the electronic coupling is essentially mediated by a single pathway or rather by the bulk of intervening protein continues to be a matter of debate. ${ }^{9,11,14}$

In addition to being a function of the protein medium, the thermodynamics and kinetics of long-range electron transfer depend on the electronic structure of the prosthetic groups involved in the reaction. ${ }^{15-18}$ Detailed information about the electronic structure of the metal clusters participating in biological electron transfer has been obtained from spectroscopic investigations. ${ }^{2,19,20}$ Conspicuous electronic features revealed by Mössbauer studies of trinuclear and tetranuclear mixed-valence $\mathrm{Fe}-\mathrm{S}$ clusters in both proteins and synthetic analogs are the delocalization of an "extra" electron over two metal sites of the cluster and the concomitant ordering of the $\mathrm{Fe}$ spins in $S \approx \frac{9}{2}$ delocalized $\left[\mathrm{Fe}^{2.5+} \mathrm{Fe}^{2.5+}\right]$ pairs. ${ }^{19,21}$ Theoretical studies have shown that partial electron delocalization and spin ordering in these clusters can be explained by considering an interplay of spin-dependent resonance interaction (double exchange) and vibronic coupling. ${ }^{22-25}$ The condition for electron delocalization over multiple metal sites is that the reorganization energy associated with electron trapping at one site of the cluster is smaller than the resonance energy obtained by delocalization of the extra electron. ${ }^{26}$ The $\left[\mathrm{Fe}_{2} \mathrm{~S}_{2}\right]^{+}$clusters in wild-type $2 \mathrm{Fe}$ ferredoxins are valence localized on the Mössbauer time scale. ${ }^{27}$ Recently, however, Mössbauer studies have shown that these clusters can also sustain a valence-delocalized state in mutants of the $2 \mathrm{Fe}$ ferredoxin from Clostridium pasteurianum $(C p))^{28,29}$ The valence-delocalized clusters are found in samples containing both valence-localized and valencedelocalized species. The ground-state spin of these clusters correlates with the metal valencies: The valence-localized cluster has spin $S=\frac{1}{2}$ (denoted $F d_{1 / 2}$ ) and the valencedelocalized cluster has $S=\frac{9}{2}\left(F d_{9 / 2}\right)$. This correlation is in agreement with the double-exchange hypothesis, viz. that resonance delocalization gives rise to ferromagnetic ordering of the metal spins. ${ }^{21,25,30-35}$ The relative amounts of $F d_{9 / 2}$ depend sensitively on the conditions under which the protein samples were prepared, which attests to the possibility that the electronic state of the cluster is susceptible to tuning by the protein environment. ${ }^{36}$ Ferromagnetic spin ordering $\left(S=\frac{9}{2}\right)$ has also been observed in the valence-delocalized complex $\left[\mathrm{Fe}_{2}(\mathrm{OH})_{3}(\mathrm{tmtacn})_{2}\right]^{2+}$ which has been identified as a class-III compound according to Robin and Day classification $^{37}$ on the basis of its spectroscopic properties. ${ }^{35,38-40}$ By extrapolating the value for the metalmetal resonance parameter in $\left[\mathrm{Fe}_{2}(\mathrm{OH})_{3}(\mathrm{tmtacn})_{2}\right]^{2+}$ to plant-type $2 \mathrm{Fe}$ ferredoxins, Gamelin et al. have inferred that the $S=\frac{9}{2}$ excited state of the latter systems is fully delocal- ized (class III). ${ }^{39}$ As the ground state of $F d_{9 / 2}$ must result from a rearrangement of the spin levels in the wild-type cluster, the $S=\frac{9}{2}$ ground state in the mutant cluster is probably class III as well. ${ }^{28}$ The studies of $\left[\mathrm{Fe}_{2}(\mathrm{OH})_{3}(\mathrm{tmtacn})_{2}\right]^{2+}$ and $\left[\mathrm{Fe}_{2} \mathrm{~S}_{2}\right]^{+}$clusters of $2 \mathrm{Fe}$ ferredoxin mutants from $C p$ corroborate the notion that mixed-valence clusters with paramagnetic sites can accommodate a set of closely spaced electronic spin states with different degrees of valence delocalization. ${ }^{15,28}$ Other examples of a correlation between valence delocalization and spin ordering can be found in recent literature on organic radicals. ${ }^{41,42}$

Valence delocalization in the initial (or final) state of an electron-transfer reaction leads to a reduction in the innersphere reorganization energy for electron abstraction (or addition). ${ }^{15,43,44}$ This reduction lowers the activation energy, $\Delta G^{*}$, and increases the rate constant for cluster-based selfexchange reactions as a function of the delocalization degree of the mixed-valence cluster. Indeed, the predicted trend is borne out by the self-exchange rates observed for a series of binuclear manganese compounds. ${ }^{15,45}$ The relationship between spin ordering and valence delocalization suggests that the cluster spin is a rate-determining factor for selfexchange.

Spin effects on electron transfer are, however, more complex than the foregoing reasoning suggests. In an earlier article we developed a semiclassical theory for long-range electron transfer from a mixed-valence $2 \mathrm{Fe}$ cluster with exchange-coupled paramagnetic sites to a diamagnetic acceptor. ${ }^{15}$ In this theory, the extra electron is considered to be the "transferable" electron. The excited spin states of $\mathrm{Fe}-\mathrm{S}$ clusters have excitation energies comparable to the thermal activation energy at physiological temperatures and, therefore, contributions to the rate constant from both ground and excited states have to be taken into account. The effective electronic coupling of donor and acceptor has a marked dependence on spin and is subject to spin selection rules. These rules imply that electron-transfer reactions occur in "spin channels." The motion (delocalization) of the extra electron depends on the spin of the state of the cluster and reflects the relative importance of spin-dependent resonance delocalization and vibronic trapping. In the initial state of the reaction, the effective potential "felt" by the extra electron embodies the concerted effects of resonance delocalization, Heisenberg-Dirac-van Vleck (HDvV) antiferromagnetic exchange, and vibronic interactions, while in the final state there are only energy contributions from the latter two interactions. ${ }^{15}$ Due to the spin dependence of the interaction energies, each spin channel has its own free-energy change and reorganization parameter from which channel-specific activation energies can be evaluated. The latter quantities determine the transfer capabilities of the individual spin channels from which the net electron-transfer rate is calculated by taking the thermal average. The net rate constant, when plotted versus the driving force $\left(-\Delta G^{0}\right)$, gives rise to a curve with a skewed bell shape. The asymmetry of this curve is due to electron transfer via excited spin levels. The contributions of the latter states are significant, especially if the minima of the activation barriers for the spin channels are dispersed over a broad range on the driving-force scale as a 
result of spin-dependent resonance interaction. In particular, excited spin states can facilitate electron transfer with a low driving force, a type of reaction often encountered in biology. In the presence of double exchange, $\mathrm{HDvV}$ exchange can tune the rate constant, suggesting that a cluster can act as a molecular switch for exchange-controlled electron gating.

The theoretical treatment given in Ref. 15 is based on the following premises: (1) There is a single effective reaction coordinate for the electron-transfer reaction; (2) the potential energy surfaces are harmonic; (3) the rate constants for the spin channels are given by Marcus' semiclassical expression (see above); (4) outer-sphere contributions to reorganization energy are negligible; and (5) the electronic matrix element for the donor-acceptor interaction is nonzero only for one of the metal sites of the cluster. Hereafter, the model formulated in Ref. 15 will be referred to as the effective mode (EM) model. In this study we investigate to what extent release of these premises may affect the results obtained with the EM model. Thus, we consider here: $\left(1^{\prime}\right)$ a multimode model for inner-sphere reorganization, taking into account three locally symmetric breathing modes, one at each metal site of the dimer and one at the acceptor; $\left(2^{\prime}\right)$ anharmonic potential surfaces, calculated by using an extension of the semiclassical treatment of mixed-valence dimers given by Piepho, Krausz, and Schatz ${ }^{26,46}$ (PKS model) to the present case of spin-dependent resonance interaction. The theoretical model which includes these extensions will be termed the semiclassical (SC) model. ( $\left.3^{\prime}\right)$ Effects on the rate constant associated with the kinetic energy of the innersphere vibrations are explicitly taken into account (tunneling). The vibronic states arising from electron-vibrational interactions along the symmetry-breaking mode of the dimer are obtained by numerical application of the quantummechanical version of the PKS model; the remaining modes are treated analytically, in the framework of the FranckCondon theory. The quantum treatment should provide more accurate results, particularly in the case that $\hbar \omega_{\text {inner }}>k_{B} T$. $\left(4^{\prime}\right)$ The influence of outer-sphere reorganization on the rate for electron transfer from donor to acceptor is evaluated in semiclassical approximation. This approach is based on the well-documented fact that outer-sphere reorganization energy originates mainly from coupling to soft modes $\left(\hbar \omega_{\text {outer }} \ll k_{B} T\right){ }^{47}$ The outer-sphere contributions to reorganization energy can then be lumped into a single reorganization parameter $\left(\chi_{\text {out }}\right)$, according to Marcus' theory. ${ }^{8}$ The evaluation of the outer-sphere reorganization effect has been combined with the quantum-mechanical description of innersphere reorganization by following a treatment given by Ulstrup and Jortner. ${ }^{48}$ The resulting QM model is the most detailed theory considered in this article. $\left(5^{\prime}\right)$ Electron transfer can occur from either metal ion of the dimer. This last extension accounts for situations in which the protein provides an efficient electronic coupling between the acceptor and the two metal sites of the dimer. Earlier studies have revealed that the multiple connectivity of the metal sites in mixed-valence clusters with a triangular or tetrahedral topology is an essential premise for the remarkable valencedelocalization properties of these systems (see above). ${ }^{22-24,49}$ For example, in an isosceles triangle, the effective electronic coupling between the apex orbital and orbital states that extend over the two remaining sites either vanishes or prevails, depending on the relative sign occurring in the linear combination of the localized orbitals, that is, $\left\langle\varphi_{3}|h| \varphi_{1}-\varphi_{2}\right\rangle$ $=0$ and $\left\langle\varphi_{3}|h| \varphi_{1}+\varphi_{2}\right\rangle \neq 0$, where $h$ is an effective oneelectron operator describing the motion of the extra electron. The rate constant for transfer to the third site is proportional to the square of the effective coupling constant $\left(H_{\mathrm{DA}}^{(1)}\right.$ $\left.\pm H_{\mathrm{DA}}^{(2)}\right)^{2}$ which is a nonadditive quantity with respect to electron-transfer pathways, where we have used the definition $H_{\mathrm{DA}}^{(i)} \equiv\left\langle\varphi_{3}|h| \varphi_{i}\right\rangle$. It is important to note that the rate constant is not proportional to the additive, phaseindependent factor $\left(H_{\mathrm{DA}}^{(1)}\right)^{2}+\left(H_{\mathrm{DA}}^{(2)}\right)^{2}$. The extent of nonadditivity and its dependence on spin will be analyzed in this study.

The protein provides the cluster with an irregular environment, which excludes symmetry elements interchanging the metal atoms of the cluster. The inequivalencies in the surroundings of the individual metal ions of a cluster give rise to differences in the electronic potentials for the extra electron at these sites. The various plausible causes for the potential differences, such as electrostatic interactions with amide groups, hydrogen bonds, strain effects, etc. ${ }^{50}$ will not be specified here for any particular example. Instead, it is assumed that the effect of site-differentiating interactions can be cast into a single asymmetry parameter $\left(\Delta E_{\mathrm{as}}\right) .{ }^{46}$ In the same vein, the electronic matrix elements are expected to differ (i.e., $H_{\mathrm{DA}}^{(1)} \neq H_{\mathrm{DA}}^{(2)}$ ) when the metal atoms of the donor cluster occupy inequivalent positions with respect to the acceptor. Thus, exposure of a symmetric cluster to the protein environment leads to asymmetry in the cluster structure and its interactions with the acceptor site. The effect of cluster asymmetry induced by the protein on the electron-transfer rate is investigated. The results of this theoretical analysis suggest that Nature's frequent use of clusters in biological electron-transfer chains may, in part, be due to the additional regulatory possibilities specific to polynuclear systems.

\section{FORMALISM}

Section II of the article is organized as follows. In Sec. II A we present the derivation of an expression for the rate constant of electron transfer $\left(k_{\mathrm{et}}\right)$ from a binuclear donor cluster with exchange-coupled paramagnetic sites to a diamagnetic mononuclear acceptor in the framework of a QM model. In Secs. II B and II C, the simplifications leading to the SC model and the EM model are specified. In these treatments it is assumed that the interactions inside donor and acceptor are strong compared to the electronic coupling between these units. This assumption allows us to ignore donor-acceptor interactions in the calculation of the vibronic states for these units and evaluate the rate constant by using Fermi Golden rule. Furthermore, the separations between the discrete inner-sphere energy levels, including tunneling-split vibronic doublets in mixed-valence donors of class II, are considered to be greater than the electronic donor-acceptor coupling. This condition excludes the possibility of quantum interference between contributions to the rate constant from different vibronic states. 


\section{A. Quantum-mechanical model}

The expression for $k_{\mathrm{et}}$ is derived in two steps. In Secs. II A 1-II A 5, we develop arguments leading to the expression for the transfer rate of an extra electron stored on a diamagnetic dimer to a diamagnetic acceptor. In Secs. II A 6-II A 7 the effects of spin are introduced.

\section{Hamiltonians for the dimer in initial and final state}

We consider the one-electron transfer reaction $D^{1} A^{0}$ $\rightarrow D^{0} A^{1}$, where $D$ is a binuclear donor and $A$ a mononuclear acceptor (see diagram).

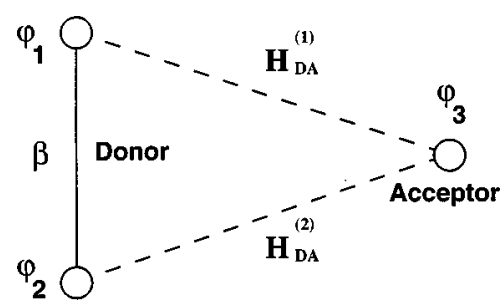

The left and right hand side of the reaction are referred to as the initial and final state. The superscripts 0 and 1 indicate the absence and presence of the extra electron at the labeled molecule, respectively. It is assumed that both the electron transfer between $D$ and $A$ as well as intracluster electron transfer between the two sites of $D$ are vibronically coupled to symmetric distortions (breathing modes) of the coordination shells of sites 1 and 2 of the dimer. ${ }^{26,46}$ These modes are denoted $q_{1}$ and $q_{2}$, respectively.

The Hamiltonian for $D$ contains operators for vibrational kinetic energy, potentials associated with elastic restoring forces (assumed to be harmonic), site energies, vibronic coupling between the extra electron and locally symmetric modes, and metal-metal resonance interaction

$$
\begin{aligned}
H_{D^{1}}= & -\frac{\hbar^{2}}{2 m_{D}} \frac{\partial^{2}}{\partial q_{1}^{2}}-\frac{\hbar^{2}}{2 m_{D}} \frac{\partial^{2}}{\partial q_{2}^{2}}+\frac{1}{2} \kappa_{D} q_{1}^{2}+\frac{1}{2} \kappa_{D} q_{2}^{2} \\
& +\Delta E_{\text {as }}\left(n_{1}-n_{2}\right)-\kappa_{D} l_{D} q_{1} n_{1}-\kappa_{D} l_{D} q_{2} n_{2} \\
& +n_{s} \beta t_{12} .
\end{aligned}
$$

The quantities occurring in Eq. (1) are defined as follows: $\kappa_{D}$ is the force constant for metal-ligand stretch, $m_{D}$ is an effective mass, $\Delta E_{\text {as }}$ is half the difference of the electronic potentials at sites 1 and $2, n_{1}$ and $n_{2}$ are the electronic occupation numbers of the two sites, and $l_{D}=\sqrt{L} \Delta R$ is a vibronic coupling parameter. In the latter expression, $L$ is the coordination number of each individual site of the dimer and $\Delta R$ is the increase in the average metal-ligand distance at a dimer site occurring upon its reduction. If the system is in the $D^{1} A^{0}$ state, the extra electron is accommodated by the localized orbitals $\varphi_{1}$ and $\varphi_{2}$ centered at sites 1 and 2 of dimer $D$. The (indirect) overlap between these orbitals gives rise to an off-diagonal metal-metal resonance interaction of strength $\beta$. The transfer operator $t_{12}$ connects the two orbital states, $\left\langle\varphi_{1}\left|t_{12}\right| \varphi_{2}\right\rangle=1$, and has eigenstates in which the extra electron is delocalized. Using the dimensionless, symmetrized coordinates, defined as

$$
x_{s, a}=\sqrt{\frac{m_{D} \omega_{D}}{\hbar}} q_{s, a}=\sqrt{\frac{m_{D} \omega_{D}}{\hbar}} \frac{q_{1} \pm q_{2}}{\sqrt{2}},
$$

the Hamiltonian can be expressed as

$$
\begin{aligned}
H_{D^{1}}= & \hbar \omega_{D}\left(-\frac{1}{2} \frac{\partial^{2}}{\partial x_{s}^{2}}-\frac{1}{2} \frac{\partial^{2}}{\partial x_{a}^{2}}+\frac{1}{2} x_{s}^{2}+\frac{1}{2} x_{a}^{2}\right. \\
& \left.+\Delta_{\text {as }} n_{a}-\lambda_{D} x_{s} n_{s}-\lambda_{D} x_{a} n_{a}\right)+n_{s} \beta t_{12},
\end{aligned}
$$

where $\omega_{D}$ is the angular frequency of the vibration which is given by

$$
\omega_{D}=\sqrt{\frac{\kappa_{D}}{m_{D}}},
$$

and the other quantities are dimensionless and defined as

$$
\begin{aligned}
& \lambda_{D}=\sqrt{\frac{m_{D} \omega_{D}}{2 \hbar}} l_{D}, \\
& \Delta_{\text {as }}=\frac{\Delta E_{\mathrm{as}}}{\hbar \omega_{D}}, \\
& n_{s, a}=n_{1} \pm n_{2} .
\end{aligned}
$$

$\lambda_{D}$ represents the vibronic coupling constant, $\Delta_{\text {as }}$ is the site energy difference expressed in vibrational quantum units, $n_{s}$ is the total electron occupation number of the dimer (also denoted as $n_{D}$ ), and $n_{a}$ is the population difference of sites 1 and 2. In the initial state of the reaction, the Hamiltonian for $D^{1}$ is obtained from Eq. (3) by taking $n_{D} \equiv n_{s}=1$ and coincides with the Hamiltonian introduced by Piepho, Krausz, and Schatz to describe the electronic-vibrational properties of mixed-valence dimers. ${ }^{26}$ The valence-localized basis states for $D^{1}$ correspond to the values $n_{a}= \pm 1$ and are represented by mutually displaced potential wells if the state energies are plotted versus $x_{a}$.

In the final state of the reaction, the extra electron occupies the localized orbital $\varphi_{3}$ at $A$ (see diagram), and the Hamiltonian for $D^{0}$ simplifies to Eq. (5) by substitution of $n_{s}=n_{a}=0$ in Eq. (3)

$$
H_{D^{0}}=\hbar \omega_{D}\left(-\frac{1}{2} \frac{\partial^{2}}{\partial x_{s}^{2}}-\frac{1}{2} \frac{\partial^{2}}{\partial x_{a}^{2}}+\frac{1}{2} x_{s}^{2}+\frac{1}{2} x_{a}^{2}\right) .
$$

Comparison of the Hamiltonians for the two oxidation states of $D$ shows that the potential wells for $D^{1}$ are displaced along $x_{s}$ over distance $\lambda_{D}$ with respect to the well for $D^{0}$.

\section{Hamiltonians for the mononuclear acceptor in initial and final state}

The breathing mode of the coordination sphere for the acceptor is denoted $q_{A}$. The vibrational Hamiltonians for the acceptor in the initial and final state can, respectively, be expressed as

$$
\begin{aligned}
& H_{A^{0}}=\hbar \omega_{A}\left(-\frac{1}{2} \frac{\partial^{2}}{\partial x_{A}^{2}}+\frac{1}{2} x_{A}^{2}\right), \\
& H_{A^{1}}=\hbar \omega_{A}\left(-\frac{1}{2} \frac{\partial^{2}}{\partial x_{A}^{2}}+\frac{1}{2} x_{A}^{2}-\lambda_{A} x_{A}\right),
\end{aligned}
$$


where we have used the dimensionless coordinate $x_{A}$ defined as

$$
x_{A}=\sqrt{\frac{m_{A} \omega_{A}}{\hbar}} q_{A},
$$

the dimensionless vibronic coupling constant,

$$
\lambda_{A}=\sqrt{\frac{m_{A} \omega_{A}}{\hbar}} l_{A},
$$

and the other symbols have analogous meanings as for $D$. The potential well of Eq. (7) is displaced along $x_{A}$ over $\lambda_{A}$ with respect to the well of Eq. (6).

\section{Eigenstates and eigenvalues for initial and final state}

The solutions for the eigenstates of the Hamiltonians for $D^{1}$ and $D^{0}$ [Eqs. (3) and (5)] have been obtained by separation of the variables $x_{s}$ and $x_{a}$. Piepho et al. ${ }^{26}$ determined solutions for the $x_{a}$-dependent part of the problem by numerical diagonalization of the electron-vibrational Hamiltonian. This procedure yields vibronic states, indicated in square brackets in Eq. (9a), which are linear combinations of valence-localized electronic states $\left(\varphi_{1}\right.$ and $\varphi_{2}$, see diagram) with $x_{a}$-dependent coefficients. The latter coefficients are expressed in a basis of Hermite polynomials $\chi_{a, k} \equiv \chi_{a, k}\left(x_{a}\right)$, centered at $x_{a}=0$. Coordinate $x_{s}$ of the dimer and breathing mode $x_{A}$ of the acceptor do not couple to intradimer electron transfer and, therefore, these variables can be factored out $\left[\chi_{s, l}^{D^{1}} \equiv \chi_{s, l}^{D^{1}}\left(x_{s}\right)\right.$ and $\chi_{m}^{A^{0}} \equiv \chi_{m}^{A^{0}}\left(x_{A}\right)$ in Eq. (9a) $]$. As a result of this factorization, the electron-vibrational eigenfunctions and energies of the initial state of the reaction $\left(D^{1} A^{0}\right)$ can be written as

$$
\begin{aligned}
\Psi_{k l m}^{D^{1} A^{0}} & =\psi_{k l}^{D^{1}} \psi_{m}^{A^{0}} \\
& =\left[\varphi_{1} \sum_{n} c_{n k}^{(1)} \chi_{a, n}^{D^{0}}+\varphi_{2} \sum_{n} c_{n k}^{(2)} \chi_{a, n}^{D^{0}}\right] \chi_{s, l}^{D^{1}} \chi_{m}^{A^{0}}, \\
E_{k l m}= & \delta+\epsilon_{k}^{P K S}+\hbar \omega_{D}\left(l+\frac{1}{2}\right)+\hbar \omega_{A}\left(m+\frac{1}{2}\right) .
\end{aligned}
$$

We assume that the force constants (and frequencies) of the vibrational modes do not change during the reaction. Thus, the antisymmetric eigen vibrations for $D^{0}$ can be chosen as $\chi_{a, n}$ basis. This choice is expressed by labeling these functions with $D^{0}$ in both the final and initial states of the reaction [see Eqs. (9a) and (10a)]. Parameter $\delta$ [Eq. (9b)] is the magnitude of the free energy change for electron transfer from $D$ to $A$ in the case that $\beta=0$ and $J=0$ (see below). The second term in Eq. (9b) is the energy eigenvalue of the vibronic state in square brackets [Eq. (9a)]. The solutions for $D^{1}$ in Eq. (9) correspond to valence delocalization of classIII type if $|\beta| /\left(\lambda_{D}^{2} \hbar \omega_{D}\right) \geqslant 1 .{ }^{46}$

The electron-vibrational eigenstates and energies for the final state of the reaction $\left(D^{0} A^{1}\right)$ are represented as

$$
\begin{aligned}
& \Psi_{k^{\prime} l^{\prime} m^{\prime}}^{D^{0} A^{1}}=\psi_{k^{\prime} l^{\prime}}^{D^{0}} \psi_{m^{\prime}}^{A^{1}}=\varphi_{3} \chi_{a, k^{\prime}}^{D^{\prime}} \chi_{s, l^{\prime}}^{D^{0}} \chi_{m^{\prime}}^{A^{1},}, \\
& E_{k^{\prime} l^{\prime} m^{\prime}}^{\prime}=\hbar \omega_{D}\left(k^{\prime}+\frac{1}{2}\right)+\hbar \omega_{D}\left(l^{\prime}+\frac{1}{2}\right)+\hbar \omega_{A}\left(m^{\prime}+\frac{1}{2}\right) .
\end{aligned}
$$

The vibrational part of Eq. (10a) is a product of three harmonic oscillator functions, one for each inner-sphere coordinate. Function $\chi_{s, l}^{D^{1}}$ is related to $\chi_{s, l}^{D^{0}}$ by a shift over dimensionless distance $\lambda_{D}$ [Eq. (4b)] along $x_{s}$, and $\chi_{m}^{A^{1}}$ is related to $\chi_{m}^{A^{0}}$ by a shift over $\lambda_{A}$ [Eq. (8b)] along $x_{A}$. We note that the factor 2 in the denominator of Eq. (4b) is lacking in Eq. (8b). This factor reflects the nuclearity of $D$ and gives rise to a reduction in the reorganization energy for electron abstraction from the dimer in the case that this unit is valence delocalized [compare also Eqs. (13b) and (13c) given below]. ${ }^{15,43}$ Thus, if we make the simplifying assumptions $m_{D}=m_{A}, \omega_{D}=\omega_{A}$, and $l_{D}=l_{A}$, the dimensionless shifts are related as $\lambda_{D}=\lambda_{A} / \sqrt{2}$.

\section{Electronic matrix element and Franck-Condon factors for inner-sphere vibrations}

In the general case, the two donor sites occupy inequivalent positions with respect to the acceptor. This leads to different values for the electronic coupling parameters for the two sites (see also diagram)

$$
\begin{aligned}
& H_{\mathrm{DA}}^{(1)} \equiv\left\langle\varphi_{1}\left|H_{e}\right| \varphi_{3}\right\rangle \equiv d_{1} H_{\mathrm{DA}}, \\
& H_{\mathrm{DA}}^{(2)} \equiv\left\langle\varphi_{2}\left|H_{e}\right| \varphi_{3}\right\rangle \equiv d_{2} H_{\mathrm{DA}},
\end{aligned}
$$

where $H_{e}$ is an electronic Hamiltonian describing the Coulomb interactions in the system and $d_{i}$ are dimensionless factors introduced to differentiate the two coupling constants. The electron-vibrational matrix elements linking the initial states with the final states of the reaction can be expanded as

$$
\begin{aligned}
& \left\langle\Psi_{k l m}^{D^{1} A^{0}}\left|H_{e}\right| \Psi_{k^{\prime} l^{\prime} m^{\prime}}^{D^{0} A^{1}}\right\rangle \\
& \quad=H_{\mathrm{DA}}\left(d_{1} c_{k^{\prime} k}^{(1)}+d_{2} c_{k^{\prime} k}^{(2)}\right)\left\langle\chi_{s, l}^{D^{1}} \mid \chi_{s, l^{\prime}}^{D^{0}}\right\rangle\left\langle\chi_{m}^{A^{0}} \mid \chi_{m^{\prime}}^{A^{1}}\right\rangle .
\end{aligned}
$$

The first factor in Eq. (12) is the global electronic matrix element; the remaining factors give rise to Franck-Condontype factors in the transition probability. The latter quantity is proportional to the square of the matrix element according to Fermi Golden rule. While the first vibrational factor in Eq. (12) is obtained by numerical diagonalization, the squares of the last two factors are given by the analytical expression

$\left\langle\chi_{n} \mid \chi_{n+p}^{s}\right\rangle^{2}=n !(n+p) ! s^{p} e^{-s}\left[\sum_{k=0}^{n} \frac{(-s)^{k}}{k !(n-k) !(p+k) !}\right]^{2}$,

where the angular vibrational frequency is assumed to be independent of oxidation state. ${ }^{48,51,52}$ Parameter $s$ depends on the coordinate shift and is given by

$$
\begin{aligned}
& s_{s}=\frac{1}{2} \lambda_{D}^{2}=\frac{m_{D} \omega_{D} l_{D}^{2}}{4 \hbar}, \\
& s_{A}=\frac{1}{2} \lambda_{A}^{2}=\frac{m_{A} \omega_{A} l_{A}^{2}}{2 \hbar} .
\end{aligned}
$$

\section{Energy conservation and outer-sphere vibrations}

Energy conservation requires coincidence of the initial and final state energies of an electron-transfer event. As the 
inner-sphere vibronic levels form a discrete spectrum, these energies will virtually never match, which would lead to a complete inhibition of the reaction. ${ }^{53}$ However, the transfer is also coupled to a bath of low-frequency modes ( $\hbar \omega_{\text {out }}$ $\left.\ll k_{B} T\right)$ associated with motions of the protein or solution medium in which $D$ and $A$ are immersed. This coupling gives rise to outer-sphere contributions to the reorganization energy which can be treated in the framework of Marcus' semiclassical theory [see Eq. (14b)]. ${ }^{8,54}$ Importantly, outer-sphere vibrations form a quasicontinuum and thus can match differences between the discrete levels of the inner sphere. Ulstrup and Jortner have shown that Marcus' treatment of the outersphere effect can be applied to the individual transitions between the discrete inner-sphere states of the $D A$ complex. ${ }^{48}$ This approach has the great advantage that the concerted effect of outer-sphere reorganization is lumped into a single parameter, $\chi_{\text {out }}$. The net rate constant is then obtained by summing the rate constants for the individual transitions, multiplied by the thermal factors of the initial states

$$
\begin{aligned}
k_{\mathrm{et}}= & \frac{2 \pi}{\hbar} \sum_{k, l, m} \frac{\exp \left(-E_{k l m} / k_{B} T\right)}{Z} \sum_{k^{\prime}, l^{\prime}, m^{\prime}} G_{\chi_{\mathrm{out}}}^{\mathrm{class}}\left(T, E_{k^{\prime} l^{\prime} m^{\prime}}^{\prime}\right. \\
& \left.-E_{k l m}\right)\left[H_{\mathrm{DA}}\left(d_{1} c_{k^{\prime} k}^{(1)}+d_{2} c_{k^{\prime} k}^{(2)}\right)\left\langle\chi_{s, l}^{D^{1}} \mid \chi_{s, l^{\prime}}^{D^{0}}\right\rangle\left\langle\chi_{m}^{A^{0}} \mid \chi_{m^{\prime}}^{A^{1}}\right\rangle\right]^{2},
\end{aligned}
$$

$$
\begin{aligned}
G_{\chi_{\text {out }}}^{\text {class }}\left(T, E_{k^{\prime} l^{\prime} m^{\prime}}^{\prime}-E_{k l m}\right)= & \left(4 \pi k_{B} T \chi_{\text {out }}\right)^{-1 / 2} \exp \left[-\left(\chi_{\text {out }}\right.\right. \\
& \left.\left.+E_{k^{\prime} l^{\prime} m^{\prime}}^{\prime}-E_{k l m}\right)^{2} / 4 k_{B} T \chi_{\text {out }}\right],
\end{aligned}
$$

where $Z$ is the partition function for the initial state, $D^{1} A^{0}$.

\section{Spin}

We now consider the case that the donor has paramagnetic sites in the initial and final state of the reaction. Interactions of the HDvV and spin-dependent resonance type couple the local spins, leading to states with different values for total spin and energy. ${ }^{32}$ Magnetic susceptibility measurements on biological and synthetic metal clusters have shown that low-lying excited spin states can be significantly populated at room temperature. ${ }^{55-57}$ The total spin of the donoracceptor system is a conserved quantity in electron-transfer reactions, because the predominant terms in the fundamental electronic Hamiltonian are independent of spin. ${ }^{58}$ Thus, the electron-transfer events can be grouped into "spin channels,",

$$
\left(S_{D}=S, S_{A}=0\right) S \rightarrow\left(S_{D}=S \pm \frac{1}{2}, S_{A}=\frac{1}{2}\right) S,
$$

where we have specified in parentheses the spins for donor and acceptor in the initial $\left(D^{1} A^{0}\right)$ and final $\left(D^{0} A^{1}\right)$ state of the reaction.

The introduction of spin involves modifications of both energetic and kinetic aspects of the reaction. In the presence of paramagnetic sites, the metal-metal resonance interaction is dependent on the total spin. Let us consider the dimer $\mathrm{Fe}^{2+}-\mathrm{Fe}^{3+}$ with high-spin sites. The effect of spin can be accounted for by substituting $-(\beta / 5)\left(S+\frac{1}{2}\right)$ for $\beta$ in the spin-independent formalism. In this case, calculation of the vibronic states for $D^{1}$ requires application of the PKS formalism for each spin state by adopting all parameters as defined above and the spin-dependent value for the resonance parameter. This procedure yields spin-dependent vibronic states with energies $\epsilon_{k}^{\mathrm{PKS}}(S)$. The energies for the initial state $D^{1} A^{0}$, including resonance energy and $\mathrm{HDvV}$ energy contributions, can be written as

$$
\begin{aligned}
{ }^{S} E_{k l m}= & \delta+\epsilon_{k}^{\mathrm{PKS}}(S)+\hbar \omega_{D}\left(l+\frac{1}{2}\right)+\hbar \omega_{A}\left(m+\frac{1}{2}\right) \\
& -J_{D^{1}} S(S+1)+\frac{99}{4} J_{D^{1}},
\end{aligned}
$$

and for the final state we find

$$
\begin{aligned}
S \pm 1 / 2 E_{k^{\prime} l^{\prime} m^{\prime}}^{\prime}= & \hbar \omega_{D}\left(k^{\prime}+\frac{1}{2}\right)+\hbar \omega_{D}\left(l^{\prime}+\frac{1}{2}\right)+\hbar \omega_{A}\left(m^{\prime}+\frac{1}{2}\right) \\
& -J_{D^{0}}\left(S \pm \frac{1}{2}\right)\left(S \pm \frac{1}{2}+1\right)+30 J_{D^{0}} .
\end{aligned}
$$

The subscript of $J$ indicates the oxidation state of the dimer to which the exchange parameter applies. Equations (16a) and (16b) apply to electron transfer occurring in channels with spin-dependent driving forces and reorganization barriers which have previously been discussed in the context of the EM model. ${ }^{15}$

An electronic state for the initial reaction state can formally be represented as

$c_{1}\left|\left[\left(S_{1}, \frac{1}{2}\right) S_{1}^{\prime}, S_{2}\right] S ; \varphi_{1}\right\rangle+c_{2}\left|\left[S_{1},\left(\frac{1}{2}, S_{2}\right) S_{2}^{\prime}\right] S ; \varphi_{2}\right\rangle$,

and the final state as

$$
\left|\left[\left(S_{1}, S_{2}\right) S+\sigma, \frac{1}{2}\right] S ; \varphi_{3}\right\rangle
$$

where $\sigma= \pm \frac{1}{2}$. Using these representations, we can determine the spin dependence of the matrix elements for the electron transfer from $D$ to $A$ by a single spin recoupling operation, provided we multiply the resulting matrix elements by (i) factor 2 to correct for the fact that there are two transferable electrons and (ii) local recoupling coefficient $\sqrt{3 / 5}$. 22 The electronic part of the matrix element which connects the initial and final state [see Eq. (12)] becomes spin dependent as can be seen from Eq. (18) which presents the squared matrix element of the transition $S \rightarrow S+\sigma$

$$
\begin{aligned}
\left(d_{1} c_{k^{\prime} k}^{(1)}+d_{2} c_{k^{\prime} k}^{(2)}\right)^{2} H_{\mathrm{DA}}^{2} \rightarrow & \frac{1}{5}\left[6+2 \sigma\left(S+\frac{1}{2}\right)\right]\left(d_{1}{ }^{S} c_{k^{\prime} k}^{(1)}\right. \\
& \left.+(-1)^{\sigma+1 / 2} d_{2}{ }^{S} c_{k^{\prime} k}^{(2)}\right)^{2} H_{\mathrm{DA}}^{2} .
\end{aligned}
$$

$(-1)^{\sigma+1 / 2}$ gives an alternating sign, depending on the value of $\sigma$. The prefices of the energies [Eqs. (16a) and (16b)] and the $c$ coefficients [Eq. (18)] indicate the spin of the state for which these numbers were obtained. The expression for the rate constant is obtained by combining Eqs. (14), (16), and (18).

\section{Expression for rate constant}

The rate constant $\left(k_{\mathrm{et}}\right)$ for electron transfer from a mixed-valence dimer with paramagnetic $\left(S=\frac{5}{2}\right.$ and 2$)$ sites to a diamagnetic mononuclear acceptor is given by 


$$
\begin{aligned}
k_{\mathrm{et}}= & \pi^{1 / 2} \hbar^{-1} H_{\mathrm{DA}}^{2} Z^{-1}\left(k_{B} T \chi_{\mathrm{out}}\right)^{-1 / 2} \sum_{S=1 / 2}^{9 / 2}(2 S+1) \\
& \times \sum_{\sigma=-1 / 2}^{1 / 2} \frac{1}{5}\left[6+2 \sigma\left(S+\frac{1}{2}\right)\right] \\
& \times \sum_{k, l, m=0}^{\infty} \exp \left(-{ }^{S} E_{k l m} / k_{B} T\right) \sum_{k^{\prime}, l^{\prime}, m^{\prime}=0}^{\infty} \exp \left[-\left(\chi_{\text {out }}\right.\right. \\
& \left.\left.+{ }^{S+\sigma} E_{k^{\prime} l^{\prime} m^{\prime}}^{\prime}-{ }^{S} E_{k l m}\right)^{2} / 4 k_{B} T \chi_{\text {out }}\right]\left(d_{1} S_{c_{k^{\prime} k}^{(1)}}\right. \\
& \left.+(-1)^{\sigma+1 / 2} d_{2}{ }^{S} c_{k^{\prime} k}^{(2)}\right)^{2}\left\langle\chi_{s, l}^{D^{1} \mid} \mid \chi_{s, l^{\prime}}^{D^{0}}\right\rangle^{2}\left\langle\chi_{m}^{A^{0}} \mid \chi_{m^{\prime}}^{A^{1}}\right\rangle^{2},
\end{aligned}
$$

where inner- and outer-sphere reorganization are treated quantum mechanically and semiclassically, respectively. The partition function in Eq. (19a) is obtained by summing over the spin-vibronic states in $D^{1} A^{0}$

$$
Z=\sum_{S=1 / 2}^{9 / 2}(2 S+1) \sum_{k, l, m=0}^{\infty} \exp \left[-{ }^{S} E_{k l m} / k_{B} T\right] .
$$

Equation (19) is referred to as the QM model. For the sake of clarity, we recapitulate the parameters of the QM model: $\hbar \omega_{D}, \hbar \omega_{A}, \lambda_{D}, \lambda_{A}, \Delta E_{\text {as }}, \chi_{\text {out }}, H_{\mathrm{DA}}, d_{1}, d_{2}, \beta, \delta, J_{D^{1}}$, $J_{D^{0}}$. Rearrangement of Eq. (19a) allows elimination of two summations and makes the expression amenable to fast evaluation by means of a personal computer. In order to reduce the number of free parameters in the numerical applications of the model, we make the assumptions that $\lambda_{D}$ $=\lambda_{A} / \sqrt{2}$ and $\hbar \omega_{D}=\hbar \omega_{A}$ throughout the rest of the article.

\section{B. Semiclassical model}

The SC model is a widely used method for describing electron-vibrational systems. In this model, the quantum character of nuclear motion is neglected. The nuclear subsystem is described not by the vibrational wave function but only by the potential energy (adiabatic potential). This approach is especially useful in the analysis of optical and radiationless (as in our case) transitions in complex systems for which efficient methods for the calculation of electronvibrational states are not available. For example, the SC method has been successfully applied in band shape analysis of optical transitions for Jahn-Teller systems. ${ }^{59}$ Marcus' theory for electron transfer was originally formulated as a SC model and only later recognized to be the SC limit of a fully quantum-mechanical theory. More recently, SC methods were used in the calculation of electron-transfer probabilities for systems in which the electron donor manifests the JahnTeller effect. ${ }^{60}$ The important advantage of the SC approach is its applicability to electronic transitions in which several local vibrational modes of the same symmetry are involved. In this case, instead of a multiple-mode treatment, a unique effective mode of the corresponding symmetry can be used for calculating the adiabatic potentials. ${ }^{59}$ As a consequence, the dimensionality of electron-vibrational problem can be significantly reduced. The SC methods are valid for high temperatures, $k_{B} T>\hbar \omega$, and strong vibronic coupling, $\lambda^{2}$ $\gg 1$.
The SC analog of the quantum-mechanical expression for the electron-transfer rate constant [Eq. (14a)] is obtained by replacing the summation over vibronic states by an integration over vibrational coordinates

$$
\begin{aligned}
k_{\mathrm{et}}= & \frac{2 \pi}{\hbar Z} \int d \mathbf{x}\left|\left\langle\Psi_{D^{1} A^{0}}(\mathbf{x})|H| \Psi_{D^{0} A^{1}}(\mathbf{x})\right\rangle\right|^{2} \\
& \times \exp \left(-E(\mathbf{x}) / k_{B} T\right) \delta\left(E(\mathbf{x})-E^{\prime}(\mathbf{x})\right),
\end{aligned}
$$

where the vector vibrational coordinate $\mathbf{x}$ represents the three vibrational coordinates of our problem $x_{s}, x_{a}$, and $x_{A}$. In most systems the electronic wave functions do not depend significantly on the vibrational coordinates. However, our case is an exception because the initial-state donor wave function has the form $c_{1}\left(x_{a}\right) \varphi_{1}+c_{2}\left(x_{a}\right) \varphi_{2}$, where the coefficients are rapidly varying functions of $x_{a}$. The squared matrix element in Eq. (20) can be written as

$$
\left|\left\langle\Psi_{D^{1} A^{0}}(\mathbf{x})|H| \Psi_{D^{0} A^{1}}(\mathbf{x})\right\rangle\right|^{2}=\left[d_{1} c_{1}\left(x_{a}\right)+d_{2} c_{2}\left(x_{a}\right)\right]^{2} H_{\mathrm{DA}}^{2} .
$$

The energy of the donor in the initial state is represented by a two-sheet adiabatic potential which is obtained by diagonalization of the Hamiltonian in Eq. (3) from which the nuclear kinetic energy is omitted. For reasonable values of the metal-metal interaction parameter $\beta$ (Ref. 39) and at room temperature, the population of the upper potential energy sheet can be ignored and only the lower sheet needs to be considered

$$
\begin{aligned}
E_{D^{1}}= & \delta+\hbar \omega_{D}\left[\frac{1}{2} x_{s}^{2}+\frac{1}{2} x_{a}^{2}-\lambda_{D} x_{s}+\frac{\Delta_{\mathrm{as}}}{2}\right. \\
& \left.-\sqrt{\beta^{2}+\left(\lambda_{D} x_{a}+\frac{\Delta_{\mathrm{as}}}{2}\right)^{2}}\right]
\end{aligned}
$$

The energy of the donor in the final state is given by

$$
E_{D^{0}}=\hbar \omega_{D}\left(\frac{1}{2} x_{s}^{2}+\frac{1}{2} x_{a}^{2}\right) .
$$

The initial- and final-state energies of the acceptor can be expressed as

$$
\begin{aligned}
& E_{A^{0}}=\hbar \omega_{A}\left(\frac{1}{2} x_{A}^{2}\right), \\
& E_{A^{1}}=\hbar \omega_{A}\left(\frac{1}{2} x_{A}^{2}-\lambda_{A} x_{A}\right) .
\end{aligned}
$$

Using the $\delta$ function for energy conservation in the integration in Eq. (20), the three-dimensional integration is reduced to a two-dimensional one.

In the case of a system with paramagnetic sites, the integration in Eq. (20) has to be extended by summation over spin states. As described in Sec. II A 6, the electronic energies include contributions for HDvV exchange. The effect of spin on the metal-metal resonance interaction is accounted for by taking $-(\beta / 5)\left(S+\frac{1}{2}\right)$ for $\beta$. The parameters for the SC model are: $\chi_{0, D}, \chi_{0, A}, \Delta E_{\mathrm{as}}, H_{\mathrm{DA}}, d_{1}, d_{2}, \beta, \delta, J_{D^{1}}, J_{D^{0}}$. Thus, the SC model does not explicitly depend on the vibrational quanta and the vibronic coupling constants, but on the inner-sphere reorganization energies $\chi_{0, D}=2 \lambda_{D}^{2} \hbar \omega_{D}$ and $\chi_{0, A}=2 \lambda_{A}^{2} \hbar \omega_{A}$. 


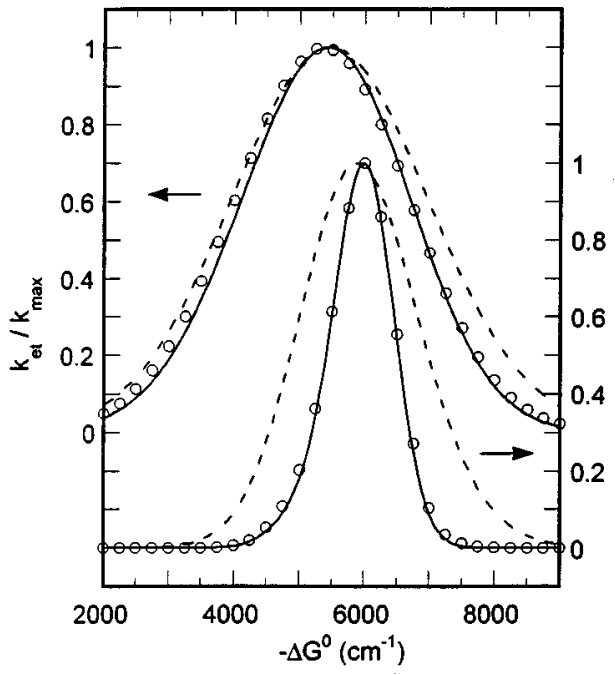

FIG. 1. Rate constant, normalized to 1 at maximum, as a function of driving force calculated with EM (-), SC (○○०), and QM (---) model at 300 $\mathrm{K}$ (upper panel) and $50 \mathrm{~K}$ (lower panel). Parameters used: $\Delta_{\text {as }}=0, d_{1}=d_{2}$ $=1, \beta=5000 \mathrm{~cm}^{-1}, J_{D^{1}}=J_{D^{0}}=-100 \mathrm{~cm}^{-1}, \hbar \omega_{D}=\hbar \omega_{A}=222 \mathrm{~cm}^{-1}, \lambda_{D}$ $=3, \lambda_{A}=4.24, \chi_{0}=4000 \mathrm{~cm}^{-1}$, and $\chi_{\text {out }}=1000 \mathrm{~cm}^{-1}$. Ground state for $D^{1}$ is class III $S=\frac{9}{2}$; the first excited state $\left(S=\frac{7}{2}\right)$ is $100 \mathrm{~cm}^{-1}$ above the ground state.

\section{Effective mode model}

The EM model has been discussed at length in an earlier publication to which we refer for details. ${ }^{15}$ In the original application of the EM model, we considered only the case $H_{\mathrm{DA}}^{(2)}=0$. Here, the EM model is extended in a straightforward manner to describe electron transfer from the two metal sites of $D$. Under the same parameter restraints as adopted for the SC model, the rate constant of the EM model depends on the following parameters: $\chi_{0}, \Delta E_{\mathrm{as}}, H_{\mathrm{DA}}, d_{1}, d_{2}$,

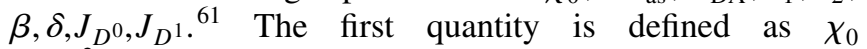
$=2 \lambda_{D}^{2} \hbar \omega_{D}$ and represents the inner-sphere reorganization parameter for electron transfer between localized species.

\section{RESULTS AND DISCUSSION}

The rate constant for electron transfer from $D$ to $A$ has been calculated as a function of $\delta$. The results have been plotted in Figs. 1-6 versus the difference $\delta-\delta_{\min }$ which is equal to the driving force $-\Delta G^{0}$. Parameter $\delta_{\min }$ is defined as the $\delta$ value for which the ground-state energies for $D^{1} A^{0}$ and $D^{0} A^{1}$ are equal, in the general case in which $\beta$ and $J$ do not vanish. ${ }^{62}$ The plots of rate constant versus driving force exhibit bell-shaped curves (Fig. 1-6). According to Marcus' theory, the maximum rate corresponds to activationless electron transfer and the regions to the left and the right of the extreme represent the normal and inverted regime, respectively. This interpretation remains valid for the individual spin channels but has to be refined when there are sizeable excited-state contributions to the electron-transfer process. The systems considered here are specified by the parameter values given in the figure captions. We have studied cases where the inner-sphere reorganization energy is greater than the outer-sphere reorganization energy, $\chi_{0}>\chi_{\text {out }} \cdot{ }^{63}$ Thus, the plots in the figures envelop essentially a manifold of discrete inner-sphere transitions between the states for $D^{1} A^{0}$

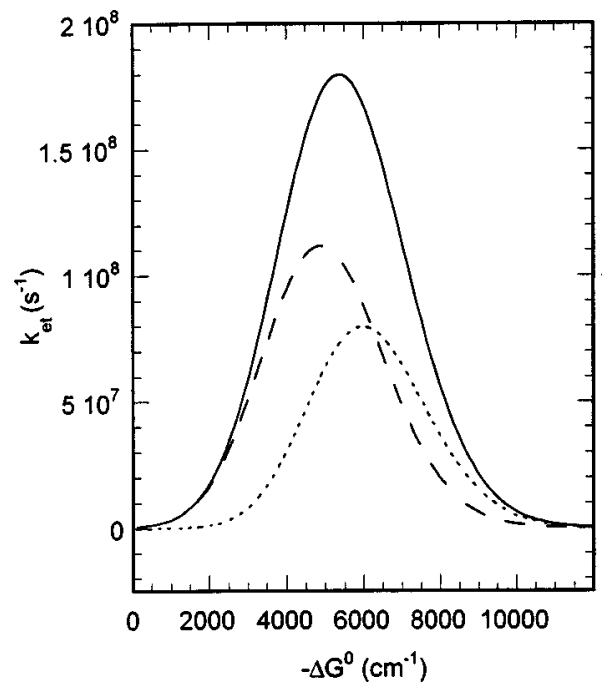

FIG. 2. Rate constant as a function of driving force calculated with QM model at $300 \mathrm{~K}(-)$. Also indicated are rates for transfer via ground (---) and excited (---) states. Parameters used: $\Delta_{\text {as }}=0, d_{1}=d_{2}=1, \beta$ $=5000 \mathrm{~cm}^{-1}, J_{D^{1}}=J_{D^{0}}=-100 \mathrm{~cm}^{-1}, \hbar \omega_{D}=\hbar \omega_{A}=500 \mathrm{~cm}^{-1}, \lambda_{D}=2, \lambda_{A}$ $=2.83, \chi_{0}=4000 \mathrm{~cm}^{-1}, \chi_{\text {out }}=1000 \mathrm{~cm}^{-1}$, and $H_{\mathrm{DA}}=1 \mathrm{~cm}^{-1}$. Ground state for $D^{1}$ is class III $S=\frac{9}{2}$; the first excited state $\left(S=\frac{7}{2}\right)$ is $100 \mathrm{~cm}^{-1}$ above the ground state.

and $D^{0} A^{1}$. For $\chi_{\text {out }}<k_{B} T$, the QM curves are resolved into a series of peaks for the individual transitions (not shown), while for larger values of $\chi_{\text {out }}$ the curves are smooth with shapes independent of the precise value for $\chi_{\text {out }}$. The effect of outer-sphere reorganization is accounted for in the formulation of the QM model, but ignored in our semiclassical treatments. In the latter two models, the outer-sphere effect on $k_{\text {et }}$ has been introduced a posteriori by shifting the $k_{\text {et }}$ versus $-\Delta G^{0}$ plots by $\chi_{\text {out }}$ in the direction of higher driving force.

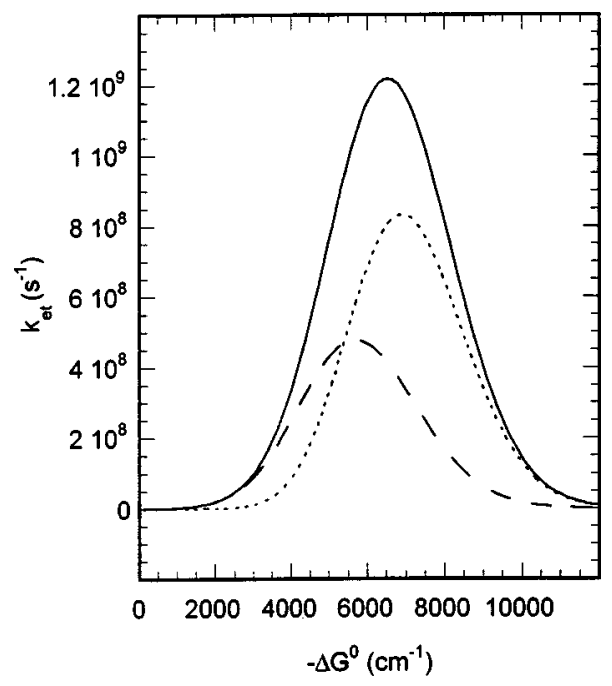

FIG. 3. Rate constant as a function of driving force calculated with QM model at $300 \mathrm{~K}(-)$. Also indicated are rates for transfer via ground (---) and excited (-- ) states. Parameters used: $\Delta_{\mathrm{as}}=0, d_{1}=d_{2}=1, \beta=$ $-5000 \mathrm{~cm}^{-1}, J_{D^{1}}=J_{D^{0}}=-100 \mathrm{~cm}^{-1}, \hbar \omega_{D}=\hbar \omega_{A}=500 \mathrm{~cm}^{-1}, \lambda_{D}=2, \lambda_{A}$ $=2.83, \chi_{0}=4000 \mathrm{~cm}^{-1}, \chi_{\text {out }}=1000 \mathrm{~cm}^{-1}$, and $H_{\mathrm{DA}}=1 \mathrm{~cm}^{-1}$. Ground state for $D^{1}$ is class III $S=\frac{9}{2}$; the first excited state $\left(S=\frac{7}{2}\right)$ is $100 \mathrm{~cm}^{-1}$ above the ground state. 


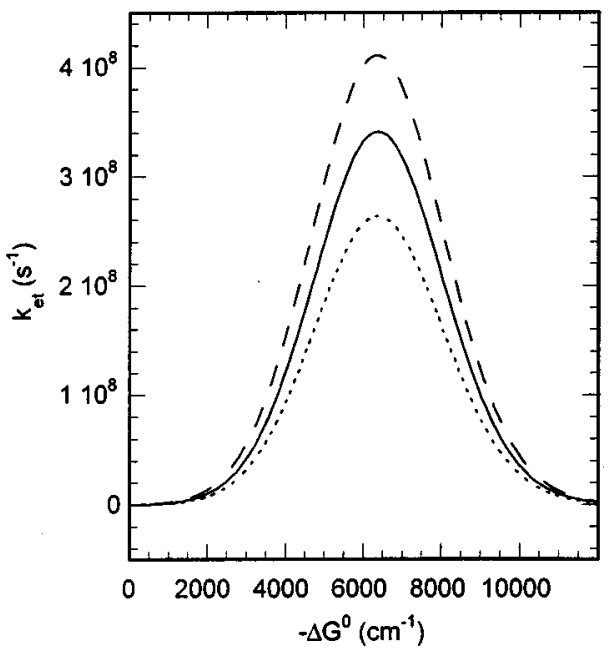

FIG. 4. Rate constant as a function of driving force calculated with QM model for $\Delta_{\text {as }}=0$ (-), $\Delta_{\text {as }}=600 \mathrm{~cm}^{-1}(---)$, and $\Delta_{\text {as }}=-600 \mathrm{~cm}^{-1}(---)$ at $300 \mathrm{~K}$. Other parameters used: $d_{1}=1, d_{2}=0, \beta=5000 \mathrm{~cm}^{-1}, J_{D^{1}}=J_{D^{0}}$ $=-100 \mathrm{~cm}^{-1}, \hbar \omega_{D}=\hbar \omega_{A}=500 \mathrm{~cm}^{-1}, \lambda_{D}=2, \lambda_{A}=2.83, \chi_{0}=4000 \mathrm{~cm}^{-1}$, $\chi_{\text {out }}=1000 \mathrm{~cm}^{-1}$, and $H_{\mathrm{DA}}=1 \mathrm{~cm}^{-1}$. Ground state for $D^{1}$ is class III $S$ $=\frac{9}{2}$; first excited state $\left(S=\frac{7}{2}\right)$ is $100 \mathrm{~cm}^{-1}$ above ground state.

\section{A. Symmetric case}

The results for symmetric systems $\left(\Delta_{\text {as }}=0\right.$ and $d_{1}=d_{2}$ $=1)$ are presented in Figs. 1-3. The system of Fig. 1 has a valence-delocalized (class-III) ground state with spin $S=\frac{9}{2}$ arising from strong resonance interaction $\left(\beta=5000 \mathrm{~cm}^{-1}\right)$. Figure 1 presents the results obtained from EM, SC, and QM calculations at two different temperatures. Comparison of the plots reveals that the results of EM and SC calculations are in excellent agreement, irrespective of temperature. The maxima in $k_{\text {et }}$ obtained from QM and from semiclassical treatments coincide (after performing the aforementioned shifts of the semiclassical results by $\chi_{\text {out }}$. By contrast, the

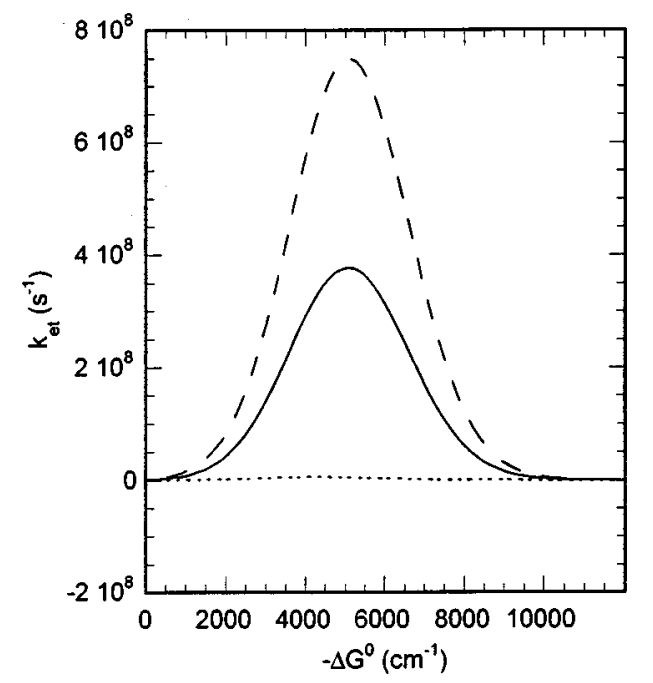

FIG. 5. Rate constant as a function of driving force calculated with QM model for $\Delta_{\text {as }}=0(-), \Delta_{\text {as }}=600 \mathrm{~cm}^{-1}(---)$, and $\Delta_{\text {as }}=-600 \mathrm{~cm}^{-1}(---)$ at $300 \mathrm{~K}$. Other parameters used: $d_{1}=1, d_{2}=0, \beta=1000 \mathrm{~cm}^{-1}, J_{D^{1}}=J_{D^{0}}$ $=-110 \mathrm{~cm}^{-1}, \hbar \omega_{D}=\hbar \omega_{A}=222 \mathrm{~cm}^{-1}, \lambda_{D}=3, \lambda_{A}=4.24, \chi_{0}=4000 \mathrm{~cm}^{-1}$, $\chi_{\text {out }}=1000 \mathrm{~cm}^{-1}$, and $H_{\mathrm{DA}}=1 \mathrm{~cm}^{-1}$. Ground state for $D^{1}$ is class II $S$ $=\frac{1}{2}$; first excited state $\left(S=\frac{3}{2}\right)$ is $300 \mathrm{~cm}^{-1}$ above ground state.

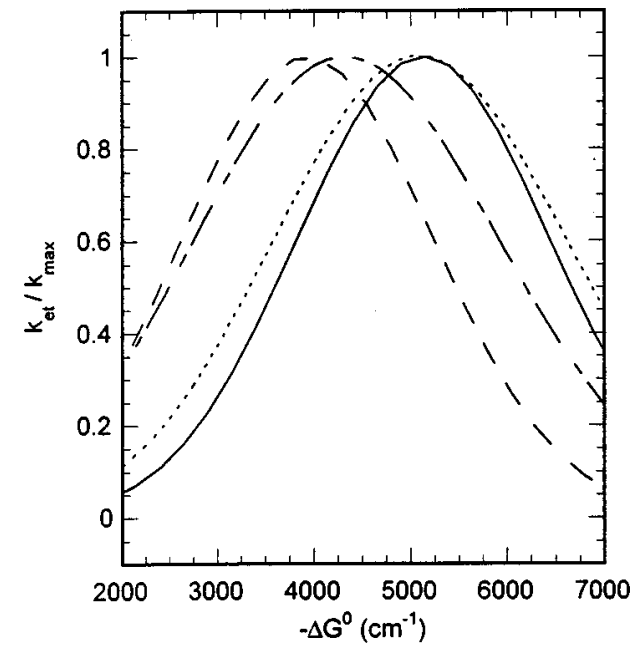

FIG. 6. Rate constant, normalized to 1 at maximum, as a function of driving force calculated with SC and QM model for $\Delta_{\text {as }}=0, \pm 600 \mathrm{~cm}^{-1}$ at $300 \mathrm{~K}$. For each model, normalized curves obtained for $\Delta_{\mathrm{as}}=0$ and $\Delta_{\text {as }}$ $=-600 \mathrm{~cm}^{-1}$ coincide: SC (-) and $\mathrm{QM}(---)$. Results for $\Delta_{\text {as }}$ $=600 \mathrm{~cm}^{-1}: \mathrm{SC}(---)$ and $\mathrm{QM}(-\cdot-\cdot)$. Other parameters used: $d_{1}=1, d_{2}$ $=0, \beta=1000 \mathrm{~cm}^{-1}, J_{D^{1}}=J_{D^{0}}=-110 \mathrm{~cm}^{-1}, \hbar \omega_{D}=\hbar \omega_{A}=222 \mathrm{~cm}^{-1}, \lambda_{D}$ $=3, \lambda_{A}=4.24, \chi_{0}=4000 \mathrm{~cm}^{-1}, \chi_{\text {out }}=1000 \mathrm{~cm}^{-1}$. Ground state for $D^{1}$ is class II $S=\frac{1}{2}$; first excited state $\left(S=\frac{3}{2}\right)$ is $300 \mathrm{~cm}^{-1}$ above ground state.

QM bell curve is wider than the EM/SC curves (see the $50 \mathrm{~K}$ curves in Fig. 1), but the curves merge at high temperature. Thus, as stated in Sec. II B, the semiclassical theory is valid in the limit $\hbar \omega_{\text {inner }} \ll k_{B} T$, whereas sizable quantum corrections occur for $\hbar \omega_{\text {inner }} \geqslant k_{B} T$. A comparison of the $300 \mathrm{~K}$ curves in Fig. 1 reveals that the QM corrections are of moderate size in the borderline region $\hbar \omega_{\text {inner }} \approx k_{B} T$. The latter result indicates that semiclassical theory provides a meaningful description of electron transfer at ambient temperatures.

In Figs. 2 and 3, we present the QM results for the net electron-transfer rate at $300 \mathrm{~K}$ and its decomposition into contributions from ground $\left(k_{\mathrm{gr}}\right)$ and excited $\left(k_{\mathrm{ex}}\right)$ spin channels. It is seen that electron transfer at low driving force occurs predominantly via the excited states. Notably, the present study corroborates one of the conclusions of a previous EM study, viz. that excited spin states are important for electron transfer involving clusters. ${ }^{15}$ The systems of Figs. 2 and 3 differ in that the sign of the resonance parameter has been reversed; the remaining parameters have the same values as in Fig. 1. Interestingly, a comparison of the two figures shows that the sign of the resonance parameter has an important effect on the electron-transfer rate. First, the rate constant for $\beta>0$ (Fig. 2) is about one order of magnitude smaller than for $\beta<0$ (Fig. 3). Second, the relative contribution of the excited states to $k_{\text {et }}$ in the former case is considerably larger than in the latter case. Third, the driving force at which $k_{\mathrm{gr}}$ is maximum for $\beta>0$ is about $10^{3} \mathrm{~cm}^{-1}$ lower than that for $\beta<0$. These results can be understood as follows. The $S=\frac{9}{2}$ electron-transfer channel is composed of two subchannels associated with the changes $\frac{9}{2} \rightarrow 4$ and $\frac{9}{2} \rightarrow 5$ of the donor spin. The transition probabilities for the two processes are proportional to $1\left(d_{1} c_{1}+d_{2} c_{2}\right)^{2} \quad\left(\frac{9}{2} \rightarrow 4\right)$ and $11\left(d_{1} c_{1}-d_{2} c_{2}\right)^{2}\left(\frac{9}{2} \rightarrow 5\right)$. In the class-III systems of Figs. 2 and 3 , the ground-state mixing coefficients are given by $c_{1}$ 
$= \pm c_{2}=1 / \sqrt{2}$, with the upper and lower sign corresponding to $\beta>0$ and $\beta<0$, respectively. Then, using $d_{1}=d_{2}=1$, we obtain the spin selection rules $\frac{9}{2} \rightarrow 4$ for $\beta>0$ and $\frac{9}{2} \rightarrow 5$ for $\beta<0$ and, accordingly, rate constants with ratio 1:11 (first point). ${ }^{64,65}$ The subchannel ratios for $S<\frac{9}{2}$ are closer to $1: 1$, which makes the rate for excited spin channels less dependent on the sign of $\beta$. As a consequence, the relative excitedstate contribution for $\beta>0$ is greater than for $\beta<0$ (second point). The shift in the position of the maxima (third point) originates from the difference $\left(-10 J^{\prime}=10^{3} \mathrm{~cm}^{-1}\right)$ in the antiferromagnetic exchange energies for the final dimer states, $S=5$ and 4 . The electronic matrix elements, connecting the donor sites with the acceptor, enter the expression for the transition probability in the combinations $\left(d_{1} c_{1} \pm d_{2} c_{2}\right)^{2}$. Disruption of the electronic coupling between one of the donor sites and the acceptor (e.g., $d_{2}=0$ ) lowers, for $\beta>0$, the transition probability of subchannel $\frac{9}{2} \rightarrow 4$, but "activates" subchannel $\frac{9}{2} \rightarrow 5$. This leads to a net increase of the rate constant by a factor of about 3 . Thus, rather surprisingly, the rate constant is enhanced by lowering the connectivity between donor and acceptor. This example illustrates how nonadditivity of electron transfer with respect to the sites of valence-delocalized polynuclear systems (see Sec. I) affects the rate constant. ${ }^{66}$ The $\beta$-sign effect on $k_{\mathrm{et}}$ is homologues to the $\beta$-sign effect on the metal valencies in symmetric $3 \mathrm{Fe}$ clusters. Thus, a small value for $k_{\text {et }}$ correlates with electron delocalization over a pair of $\mathrm{Fe}$ sites $(\beta>0)$ while a large value for $k_{\text {et }}$ correlates with complete delocalization over the three $\mathrm{Fe}$ sites of the cluster $(\beta<0)$.

\section{B. Asymmetric case}

We now consider asymmetric systems in which the electronic matrix elements for the donor-acceptor interactions are site dependent; in particular, we take $d_{1}=1, d_{2}=0$. Figures 4-6 show the effect of asymmetry parameter $\Delta_{\text {as }}$ on the rate constant of such systems. The system of Fig. 4 has strong resonance interaction, with a $\beta$ parameter equal to that of Fig. 1. Figure 4 indicates that the rate constant is rather insensitive to $\Delta_{\text {as }}$ for class-III systems, provided that $\Delta_{\text {as }}$ $<|\beta|$. The latter condition ensures that the localizing interaction of the asymmetry operator $\Delta_{\text {as }}\left(n_{1}-n_{2}\right)$ is not strong enough to disrupt the delocalized states, $\varphi_{1} \pm \varphi_{2}$. Moreover, since the expectation value of the asymmetry operator in the delocalized state is identical to zero, it follows that neither energy nor wave function is significantly affected by $\Delta_{\text {as }}$. Hence, the rate constant is not very sensitive to changes in this parameter either. Figures 5 and 6 present the QM results obtained after a fivefold reduction of the resonance parameter of Fig. 4, as a consequence of which the ground state is class-II valence localized with spin $S=\frac{1}{2}$. It can be seen from Fig. 5 that the rate constant depends sensitively on both the sign and magnitude of $\Delta_{\text {as }}$. For $\Delta_{\text {as }}<0$, the rate constant increases as a function of $\left|\Delta_{\text {as }}\right|$ by a factor independent of the driving force, and reaches the limit $k_{\mathrm{et}}\left(\Delta_{\mathrm{as}}<0\right) \approx 2 k_{\mathrm{et}}\left(\Delta_{\text {as }}\right.$ $=0$ ) for $\left|2 \Delta_{\text {as }}\right| \gg k_{B} T$. For $\Delta_{\text {as }}>0$, (i) the rate constant decreases as a function of $\Delta_{\text {as }}$ and approaches the limit $k_{\text {et }}$ $\left(\Delta_{\text {as }}>0\right)=0$ for $2 \Delta_{\text {as }} \gg k_{B} T$ and (ii) the bell-shaped curve undergoes a rigid shift to a lower driving force $\left(\approx 2 \Delta_{\text {as }}\right.$ for small values of $\beta$ ). These numerical results can be under-

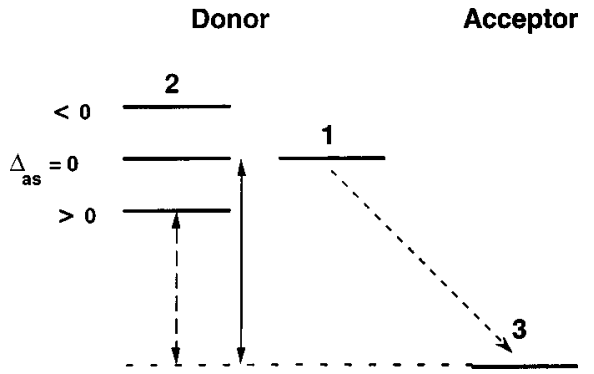

FIG. 7. Schematic level diagram representing the localized states at sites 1 and 2 of valence-localized donor and at site 3 of acceptor. Level for site 2 is indicated for three different values of $\Delta_{\text {as }}$. Electron transfer from site 1 to site 3 is indicated by a broken arrow; the transfer from site 2 to site 3 is assumed to be zero $\left(d_{2}=0\right)$. Solid and broken double arrows give the driving forces for $\Delta_{\text {as }} \leqslant 0$ and $\Delta_{\text {as }}>0$, respectively.

stood by considering Fig. 7 which depicts the level energies for sites 1 and 2 of the donor and site 3 of the acceptor. The energy of site 2 has been indicated for three different values of $\Delta_{\text {as }}$. For each value of $\Delta_{\text {as }}$, the energy for site 1 has been kept constant by an appropriate choice of the value for $\delta$. As we are dealing with a class-II system, the electron transfer can only take place if the electron occupies the localized state at site 1 (see broken arrow). Assuming that the relaxation process $2 \leftrightarrow 1$ between the two dimer sites is fast, the transfer process $1 \rightarrow 3$ is the rate limiting step of the reaction. Thus, the shape of the $k_{\mathrm{et}}$ versus $-\Delta G^{0}$ curve is determined by the latter process and independent of $\Delta_{\text {as }}$. The curve is weighed, however, by the Boltzmann factor for the thermal occupation of site 1 , a factor which is independent of driving force. For $\Delta_{\text {as }}=0$, the occupation of site 1 is $50 \%$ (Fig. 5, middle curve); for $\Delta_{\text {as }}<0$ and $\Delta_{\text {as }}>0$, the occupation of site 1 is $\approx 100 \%$ (upper curve) and $\approx 0 \%$ (lower curve), respectively, provided $\left|2 \Delta_{\text {as }}\right|>k_{B} T$. The driving force of the reaction, of course, is defined as the energy difference between the lowest levels at donor and acceptor. For $\Delta_{\text {as }}<0$, the driving force is the same as for $\Delta_{\mathrm{as}}=0$ (solid double arrow in Fig. 7) and, consequently, the position of the electrontransfer curve is not altered by asymmetry. For $\Delta_{\text {as }}>0$, however, the driving force is lowered (broken double arrow), leading to a rigid shift of the electron-transfer curve to the left as shown in Fig. 6. The size of the shift depends on the method employed to calculate its value. Thus, the QM model yields a smaller value than the SC model, as can be seen by comparing the normalized curves obtained by the two methods (see Fig. 6). The deviation is most significant in the transition range between class II and class III (i.e., $\chi_{0} / 2$ $\leqslant 2|\beta| / 5 \leqslant \chi_{0}$ for $S=\frac{1}{2}$ ), indicating that in the QM description the system exhibits already class III-like behavior in a regime where according to the SC description the system belongs to class $\mathrm{II} .{ }^{67}$ We have verified that the QM shift approaches the SC value for this quantity $\left(\approx 2 \Delta_{\text {as }}\right)$ in the limit of weak resonance interaction, $|\beta| / \chi_{0} \ll 1$.

In summary, this theoretical study shows that metal clusters have unique long-range electron-transfer properties which depend on a subtle interplay of intracluster, proteincluster, and intercluster interactions (the latter being mediated by the protein). There is (1) a capacity to transfer electrons at low driving force by making use of processes that 
proceed via excited spin channels, (2) nonadditivity of the rate constant for electron transfer from a class-III mixedvalence dimer with respect to the electronic couplings between each individual metal site of the donor and the acceptor, and (3) tuning of the rate constant for electron transfer from class-II mixed-valence dimers by means of (possibly protein-induced) differences in the electronic potentials at the two metal sites. A qualitative extrapolation of these results to higher nuclearity clusters, leads to the following predictions: $\left(1^{\prime}\right)$ electron transfer via excited spin states is greater for clusters with more than two paramagnetic metal sites because they have a considerably higher density of spin states than binuclear clusters; $\left(2^{\prime}\right)$ intricate nonadditivity effects occur as multiple connectivities are present between higher-nuclearity donor and acceptor clusters; and $\left(3^{\prime}\right)$ in contrast to the case for binuclear clusters, electron transfer from trinuclear and tetranuclear clusters (e.g., those of $\mathrm{Fe}-\mathrm{S}$ type) is also tunable by local potential differences in the presence of strong resonance interactions, provided $\beta>0$. This prediction is based on the fact that in these systems the extra electron is locked into one of several possible pairdelocalized states.

\section{ACKNOWLEDGMENT}

The article is dedicated to Professor Eckard Münck on the occasion of his 60th birthday. This research was supported by NSF Grant No. MCB 9406224.

${ }^{1}$ Electron Transfer in Inorganic, Organic, and Biological Systems, edited by J. R. Bolton, N. Mataga, and G. McLendon (American Chemical Society, Washington, DC, 1991), Vol. 228.

${ }^{2}$ R. H. Holm, P. Kennepohl, and E. I. Solomon, Chem. Rev. 96, 2239 (1996).

${ }^{3}$ G. R. Moore and G. W. Pettigrew, Cytochromes c (Springer, New York, 1990).

${ }^{4}$ Iron-Sulfur Proteins, edited by R. Cammack (Academic, San Diego, 1992), Vol. 38.

${ }^{5}$ G. Ullmann and N. M. Kostic, J. Am. Chem. Soc. 117, 4766 (1995).

${ }^{6}$ J. B. Howard and D. C. Rees, Chem. Rev. 96, 2965 (1996).

${ }^{7}$ Electron Transfer in Biology and the Solid State, edited by M. K. Johnson, R. B. King, D. M. Kurz, C. Kutal, M. L. Norton, and R. A. Scott (American Chemical Society, Washington, DC, 1990), Vol. 226.

${ }^{8}$ R. A. Marcus and N. Sutin, Biochim. Biophys. Acta 811, 265 (1985).

${ }^{9}$ C. C. Moser, J. M. Keske, K. Warncke, R. S. Farid, and P. L. Dutton, Nature (London) 355, 796 (1992).

${ }^{10}$ J. R. Winkler and H. B. Gray, Chem. Rev. 92, 369 (1992).

${ }^{11}$ C. C. Moser and P. L. Dutton, in Protein Electron Transfer, edited by D. S. Bendall (BIOS Scientific, Oxford, 1996), pp. 1-21.

${ }^{12}$ M. D. Newton, Chem. Rev. 91, 767 (1991).

${ }^{13}$ P. W. Anderson, in Magnetism, edited by G. T. Rado and H. Suhl (Academic, New York, 1963), Vol. I, p. 25.

${ }^{14}$ D. N. Beratan, J. N. Onuchic, J. R. Winkler, and H. B. Gray, Science 258, 1740 (1992).

${ }^{15}$ E. L. Bominaar, C. Achim, S. A. Borshch, J.-J. Girerd, and E. Münck, Inorg. Chem. 36, 3689 (1997).

${ }^{16}$ E. I. Solomon and M. D. Lowery, Science 259, 1575 (1993).

${ }^{17}$ J.-M. Mouesca, J. L. Chen, L. Noodleman, D. Bashford, and D. A. Case, J. Am. Chem. Soc. 116, 11898 (1994).

${ }^{18}$ J. P. Kirby, B. T. Weldon, and J. K. McCusker, Inorg. Chem. 37, 3658 (1998).

${ }^{19}$ P. Middleton, D. P. E. Dickson, C. E. Johnson, and J. D. Rush, Eur. J. Biochem. 88, 135 (1978).

${ }^{20}$ E. Münck, V. Papaefthymiou, K. K. Surerus, and J.-J. Girerd, in Metal Clusters in Proteins, edited by L. Que (American Chemical Society, Washington, D.C., 1988), Vol. 372, pp. 302-325.
${ }^{21}$ V. Papaefthymiou, J.-J. Girerd, I. Moura, J. J. G. Moura, and E. Münck, J. Am. Chem. Soc. 109, 4703 (1987).

${ }^{22}$ S. A. Borshch, E. L. Bominaar, G. Blondin, and J.-J. Girerd, J. Am. Chem. Soc. 115, 5155 (1993).

${ }^{23}$ E. L. Bominaar, S. A. Borshch, and J.-J. Girerd, J. Am. Chem. Soc. 116, 5362 (1994).

${ }^{24}$ S. A. Borshch and L. F. Chibotaru, Chem. Phys. 135, 375 (1989).

${ }^{25}$ J.-J. Girerd, J. Chem. Phys. 79, 1766 (1983).

${ }^{26}$ S. B. Piepho, E. R. Krausz, and P. N. Schatz, J. Am. Chem. Soc. 100, 2996 (1978)

${ }^{27}$ H. Beinert, R. H. Holm, and E. Münck, Science 227, 609 (1997).

${ }^{28}$ C. Achim, M. P. Golinelli, E. L. Bominaar, J. Meyer, and E. Münck, J. Am. Chem. Soc. 118, 8168 (1996).

${ }^{29}$ B. R. Crouse, J. Meyer, and M. K. Johnson, J. Am. Chem. Soc. 117, 9612 (1995).

${ }^{30}$ C. Zener, Phys. Rev. 82, 403 (1951).

${ }^{31}$ P. W. Anderson and H. Hasegawa, Phys. Rev. 100, 675 (1955).

${ }^{32}$ G. Blondin and J.-J. Girerd, Chem. Rev. 90, 1359 (1990).

${ }^{33}$ I. B. Bersuker and S. A. Borshch, Adv. Chem. Phys. LXXXI, 703 (1992).

${ }^{34}$ L. Noodleman and E. J. Baerends, J. Am. Chem. Soc. 106, 2316 (1984).

${ }^{35}$ X.-Q. Ding, E. L. Bominaar, E. Bill, H. Winkler, A. X. Trautwein, S. Drüeke, P. Chaudhuri, and K. Wieghardt, J. Chem. Phys. 92, 178 (1990).

${ }^{36}$ C. Achim, E. L. Bominaar, J. Meyer, J. Peterson, and E. Münck, J. Am. Chem. Soc. (in print).

${ }^{37}$ M. B. Robin and P. Day, Adv. Inorg. Chem. Radiochem. 10, 247 (1967).

${ }^{38}$ D. R. Gamelin, E. L. Bominaar, C. Mathonière, M. L. Kirk, K. Wieghardt, J.-J. Girerd, and E. I. Solomon, Inorg. Chem. 35, 4323 (1996).

${ }^{39}$ D. R. Gamelin, E. L. Bominaar, M. L. Kirk, K. Wieghardt, and E. I. Solomon, J. Am. Chem. Soc. 118, 8085 (1996).

${ }^{40}$ G. Peng, J. van Elp, H. Jang, L. Que, W. H. Armstrong, and S. P. Cramer, J. Am. Chem. Soc. 117, 2515 (1995).

${ }^{41}$ T. Sugawara and A. Izuoka, Mol. Cryst. Liq. Cryst. Sci. Technol., Sect. A 305, 41 (1997).

${ }^{42}$ A. Izuoka, J. Tanabe, T. Sugawara, Y. Kawada, R. Kumai, A. Asamitsu, and Y. Tokura, Mol. Cryst. Liq. Cryst. Sci. Technol., Sect. A 306, 265 (1997).

${ }^{43}$ C. Achim, E. L. Bominaar, and E. Münck, J. Biol. Inorg. Chem. 3, 126 (1998).

${ }^{44}$ S. Larsson, B. Källebring, P. Wittung, and B. G. Malmström, Proc. Natl. Acad. Sci. USA 92, 7167 (1995).

${ }^{45}$ M. M. Monzyk and R. A. Holwerda, Inorg. Chem. 31, 1969 (1992).

${ }^{46}$ K. Y. Wong and P. N. Schatz, Progr. Inorg. Chem. 28, 369 (1981).

${ }^{47}$ G. Basu, A. Kitao, and N. Go, J. Phys. Chem. B 102, 2076 (1998).

${ }^{48}$ J. Ulstrup and J. Jortner, J. Chem. Phys. 63, 4358 (1975).

${ }^{49}$ E. L. Bominaar, Z. Hu, E. Münck, J.-J. Girerd, and S. A. Borshch, J. Am. Chem. Soc. 117, 6976 (1995).

${ }^{50}$ P. J. Stephens, D. R. Jollie, and A. Warshel, Chem. Rev. 96, 2491 (1996).

${ }^{51}$ C. Manneback, Physica (The Hague) 17, 1001 (1951).

${ }^{52}$ D. DeVault, Quantum-Mechanical Tunneling in Biological Systems (Cambridge University Press, Cambridge, 1984).

${ }^{53} \mathrm{We}$ ignore here the natural linewidth.

${ }^{54}$ R. A. Marcus, J. Chem. Phys. 24, 979 (1956).

${ }^{55}$ S. J. Yoo, Z. Hu, C. Goh, E. L. Bominaar, R. H. Holm, and E. Münck, J. Am. Chem. Soc. 119, 8732 (1997).

${ }^{56}$ C. Goh, A. Nivorozhkin, S. J. Yoo, E. L. Bominaar, E. Münck, and R. H. Holm, Inorg. Chem. 37, 2926 (1998).

${ }^{57}$ G. Palmer, W. R. Dunham, J. A. Fee, R. H. Sands, T. Iizuka, and T. Yonetani, Biochim. Biophys. Acta 245, 201 (1971).

${ }^{58} \mathrm{We}$ have ignored effects of spin-orbit coupling on the electronic matrix element.

${ }^{59}$ I. B. Bersuker and V. Z. Polinger, Vibronic Interactions in Molecules and Crystals (Springer, Berlin, 1989).

${ }^{60}$ B. G. Vekhter and M. L. Rafalovich, Chem. Phys. 21, 21 (1977).

${ }^{61}$ Parameter $\lambda$ defined in Ref. 15 is a dimensional quantity and differs from the dimensionless parameters in Eqs. (4b) and ( $8 b$ ) where we have used the definition given by PKS. Furthermore, $\delta=\delta^{\prime}+\left(\chi_{0} / 4\right)$, where $\delta^{\prime}$ is the $\delta$ parameter defined in Ref. 15 .

${ }^{62}$ It is more precise to define $\delta_{\text {min }}$ as the value for which the thermal equilibrium populations of $D^{1} A^{0}$ and $D^{0} A^{1}$ are equal. In this definition, $\delta_{\min }$ is a temperature-dependent quantity. However, in the examples considered 
here the changes in $\delta_{\min }$ with temperature are only a few hundred wave numbers and, therefore, $\delta_{\min }$ is kept fixed to the low-temperature value. ${ }^{63}$ J. Jortner, J. Chem. Phys. 64, 4860 (1976).

${ }^{64}$ The order is reversed if $d_{1}$ and $d_{2}$ have opposite signs.

${ }^{65}$ The ratio approaches zero in the limit of infinite dimer spin.
${ }^{66}$ In contrast, electron transfer from class-II dimers is nearly additive with respect to the sites.

${ }^{67}$ As pointed out by Wong and Schatz (Ref. 46), Robin and Day classification of mixed-valence dimers is essentially based on a semiclassical description of these systems and classifies the transition in an abrupt manner. 